\title{
S-charl, eine ehemalige Bergbausiedlung im Unterengadin
}

Peter Abt

\section{Einleitung}

Ende Juni 1972 führte eine Gymnasialklasse der Aargauischen Kantonsschule Baden anläßlich einer Arbeitswoche landschaftskundliche Beobachtungen in S-charl durch. Unser Hauptinteresse lag bei den ehemaligen Silber- und Bleibergwerken mit den dazugehörenden Schmelzanlagen. Außerdem versuchten wir, den Kulturlandschaftswandel nach dem Abgang des Bergbaus bis zum heutigen Zeitpunkt zu verfolgen. Wir legen hier einige Auszüge aus den Berichten der einzelnen Arbeitsgruppen vor.

S-charl, eine Fraktion von Schuls, liegt in $1810 \mathrm{~m}$ Höhe an der Einmündung des Val Sesvenna ins Clemgiatal, in unmittelbarer Nähe des Nationalparks (LK Blatt 1219 S-charl). Heute stellen Sommertourismus und Viehsömmerung die wirtschaftliche Basis des Örtchens dar, im Winter ziehen die Leute von S-charl nach Schuls, da das Clemgiatal sehr lawinengefährdet ist.

\section{Geschichte}

Im Jahre 1095 wird S-charl in der Schenkungsurkunde der Herren von Tarasp an das eben gegründete Kloster Schuls als "alpes in Scharles» erstmals erwähnt. Die älteste Urkunde über die Bergwerke stammt aus dem Jahr 1317. Es sind zunächst die Tirolischen Landesfürsten, welche das Bergregal im Unterengadin innehaben. Romanische Bergbaunamen wie Mingèr, Sesvenna, Foraz usw. weisen jedoch auf eine vortirolische Bergbauperiode hin. Das 16. Jahrhundert ist eine Zeit ständiger Auseinandersetzungen zwischen dem österreichischen S-charl und dem bündnerischen Schuls. Trotzdem blühte der Bergbau, 1576 standen in S-charl 45 Häuser. 1652 ging dann das Bergregal auf Schuls über, der Bergbau verlor an Bedeutung und 1684 zählte man nur noch 16 Häuser. Die letzte Bergbauperiode unter Landammann Hans Hitz aus Klosters begann 1811. Sie erreichte 1825 einen Höhepunkt. Damals waren 70-80 Knappen in S-charl. Doch bereits 1828 wurden die Bergwerke stillgelegt. 1902 existierten noch 13 Häuser. Nach dem 2. Weltkrieg erfolgte die Güterzusammenlegung. Ende der fünfziger Jahre bekam S-charl durch den Tourismus neuen Aufschwung. Die Straße nach Schuls wurde 1963 im Zusammenhang mit Kraftwerkbauten befahrbar gemacht. 1972 zählten wir 5 Pensionen, 10 Ferienhäuser und 7 Wohnhäuser.

\section{Ackerbaurelikte}

Wie an vielen anderen Orten im Engadin läßt sich auch in S-charl der frühere Ackerbau mühelos nachweisen. Am nordwestlich des Dörfchens gelegenen Sonnenhang, in der Flur "Ils Chomps», zeichnen sich deutliche Ackerterrassen ab, die heute als Wiese oder Weide genutzt werden (Abb. 1). Die Äckerlein reichen von rund 1800 m bis 1900 m, angebaut wurden in erster Linie Gerste und Kartoffeln. Möglicherweise wurde auch im Talboden gegen "Schmelzra» Ackerbau getrieben.

Südwestlich der Ortschaft, unmittelbar an der Clemgia gelegen, erkennt man die Überreste der Mühle von S-charl (Abb. 2). Erhalten sind außer den Grundmauern noch drei Mühlsteine aus Augengneis. Der Antrieb der Mühle erfolgte vermutlich über ein oberschlächtiges Wasserrad, die Wasserzuleitung läßt sich nur schwer rekonstruieren. Wie unser Grundriß zeigt (Fig. 1), muß es sich um ein recht stattliches Gebäude von ca. $10 \times 6 \mathrm{~m}$ gehandelt haben. Über die Umstände der Wüstlegung konnten wir nichts erfahren.

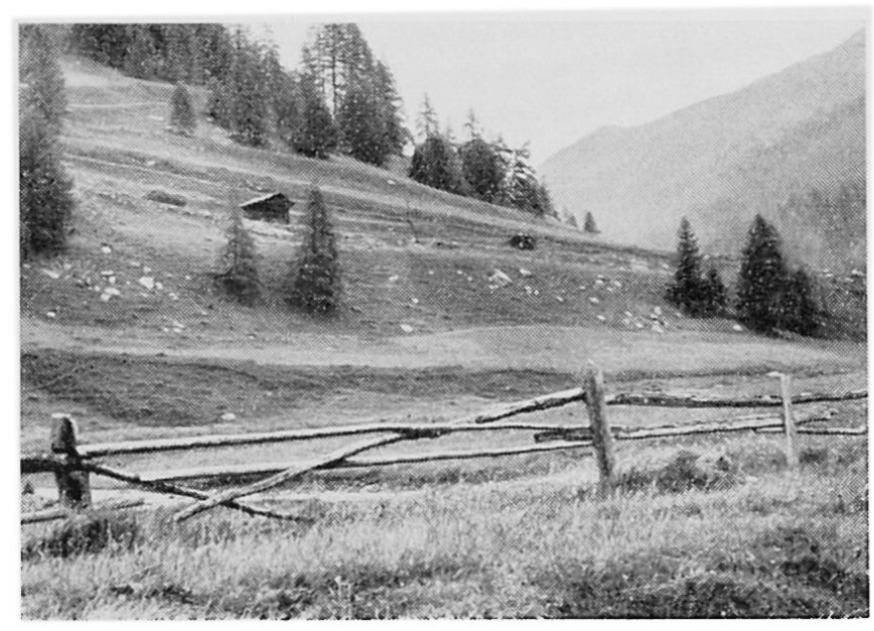

Abbildung 1. Ackerterrassen in der Flur "Ils Chomps» 


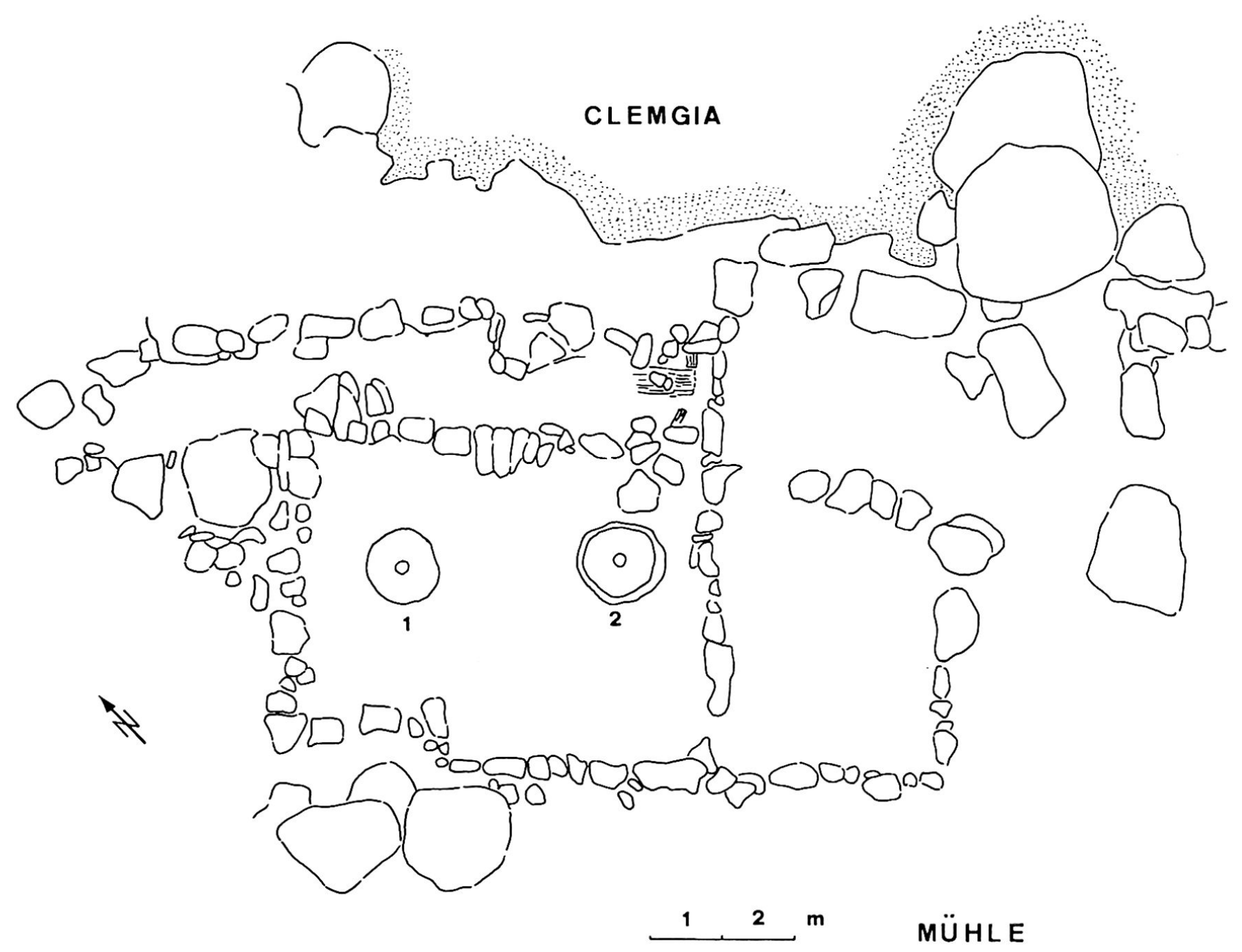

Figur 1. Steingerechte Zeichnung der Grundmauern. 1, 2: Mühlsteine

\section{Metallgewinnung}

Kohlenmeiler

Bekanntlich war früher der Bedarf an Holzkohle zur Metallgewinnung enorm groß. Wie aus der Fig. $2 \mathrm{zu}$ ersehen ist, lagen die Kohlenmeiler in der näheren Umgebung von S-charl. Man hat offensichtlich das geschlagene $\mathrm{Holz}$ an topographisch und verkehrstechnisch geeigneten Orten zu Kohle verarbeitet und nachher der Schmelze zugeführt. Sicher haben wir nicht alle Meiler erfaßt, sondern in erster Linie diejenigen, welche an den begangenen Wegen durch intensive Bodenverfärbungen auffallen. Abseits liegende Kohlenmeiler dürften inzwischen auch von Schutt überdeckt sein.

\section{Bergwerke}

Die Erze in der Umgebung von S-charl sind vor allem blei- und silberhaltig, eine genaue Beschreibung gibt Kellerhals (1962). Wir haben uns mit dem nächstgelegenen und zugleich wichtigsten $\mathrm{Ab}$ - baugebiet von Ober- und Untermadlein beschäftigt. Dabei schien es uns zweckmäßig, die dem Zerfall preisgegebenen UUbertaganlagen, welche unseres Wissens noch nirgends beschrieben wurden, herauszugreifen.

In Untermadlein, am Fuß einer großen Abraumhalde, stehen die Ruinen eines Gebäudes (Koord. $820.950 / 178.250,1850 \mathrm{~m}$ ), welches vermutlich als Unterkunft für die Knappen gedient kat. Das Haus ist massiv gebaut (vgl. Grundriß Fig. 3), vermutlich zweistöckig. Beim kleinen Raum im Erdgeschoß handelt es sich wohl um die Küche. An Wasser fehlte es in der nächsten Umgebung nicht, wird doch der wichtigste Stollen von Untermadlein heute als Wasserfassung für S-charl verwendet. Der recht gute Erhaltungszustand des Verputzes läßt die Vermutung zu, das Haus sei auch in der Bergbauperiode unter Hitz noch verwendet worden. Sicher ist jedenfalls, daß einzelne Stollen in Untermadlein durch Sprengung erweitert wurden, was den Abbau im 19. Jahrhundert belegt.

Obermadlein war das wichtigste Abbaugebiet. Auf 


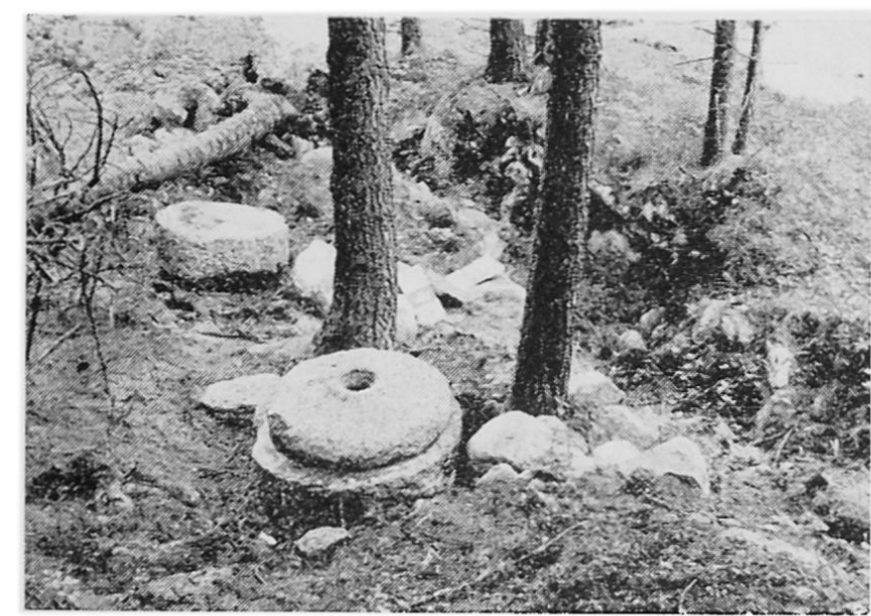

Abbildung 2. Mühle von S-charl

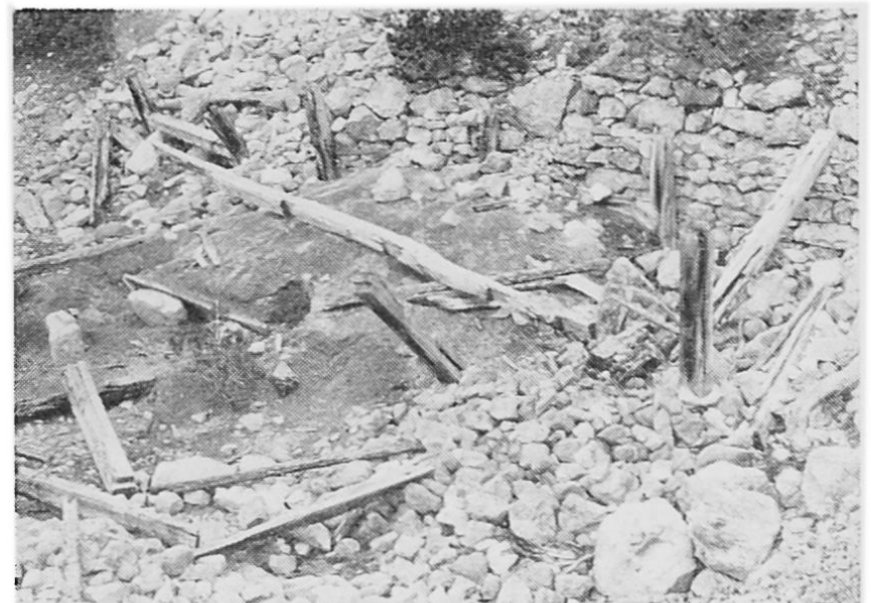

Abbildung 3. Übertaganlagen in Obermadlein, $2150 \mathrm{~m}$ rund $2150 \mathrm{~m}$ Höhe liegt das weitverzweigte Stollensystem, das von Kellerhals vermessen wurde (,Las Minieras', Koord. 821.000/179.000). Im Bereich der Stolleneingänge und der imposanten Abraumhalden erkennt man weitere zerfallende Übertaganlagen, welche am ehesten als Pochwerke und Unterkünfte für die Knappen zu interpretieren sind (Abb. 3). Der Erzabbau erfolgte nicht nur im Sommer, sondern der Wasserverhältnisse wegen besonders im Winter. So war es sicher zweckmäßig, in unmittelbarer Nähe Unterkünfte zur Verfügung zu haben. Wir dürfen auch annehmen, daß die Erze früher direkt bei den Minen durch Pochen ein erstes Mal aufbereitet wurden, die Bezeichnung «Val del Poch» wäre ein Indiz dafür. Der Erhaltungszustand dieser Anlagen ist schlecht, da es sich um Holzkonstruktionen handelt. Auf einer Fläche von $10 \times 35 \mathrm{~m}$ mit Kopfsteinpflaster befinden sich 2 gleich große Pfostenkonstruktionen von $3 \times 8,4 \mathrm{~m}$ sowie eine Art Verschlag von 2,1 x 2,1 m. Letzterer könnte als Erzsilo gedient haben, erstere vielleicht als Unterkünfte und Pochwerk. Zahlreiche Fragen im Zusammenhang mit diesen Anlagen sind noch offen, insbesondere auch die, ob sich hier oben in frühester Zeit eine Schmelzanlage befunden habe.

\section{Schmelzanlagen}

Wie aus der Fig. 2 hervorgeht, war das Gebiet von «Schmelzra» (Koord. 820.750/178.100) nicht der einzige Verhüttungsplatz, aufgrund weiterer Schlakkenhalden müssen noch mehr Schmelzanlagen bestanden haben. Wir beschränken uns im folgenden auf die Ruinen von "Schmelzra». Hier wurden die Erze in fünf Arbeitsgängen verhüttet (vgl. Peer, 1970): 1. Zerkleinern im Pochwerk, 2. Waschen in Trögen, 3. Rösten im Flammofen, 4. Reduktion im Schmelzofen, 5. Entfernen des Bleis durch «Trei- ben" im Treibherd. All diese Prozesse wurden in der letzten Phase der Metallgewinnung unter Hitz in den heute noch bestehenden Ruinen von «Schmelzra» durchgeführt. Wir vermuten indessen, daß die Gebäude mindestens teilweise aus früheren Bergbauepochen stammen. Die nachfolgende Beschreibung stützt sich auf Angaben von Herrn G. Peer, der auch den Grundriß (Fig. 4) aufgenommen hat.

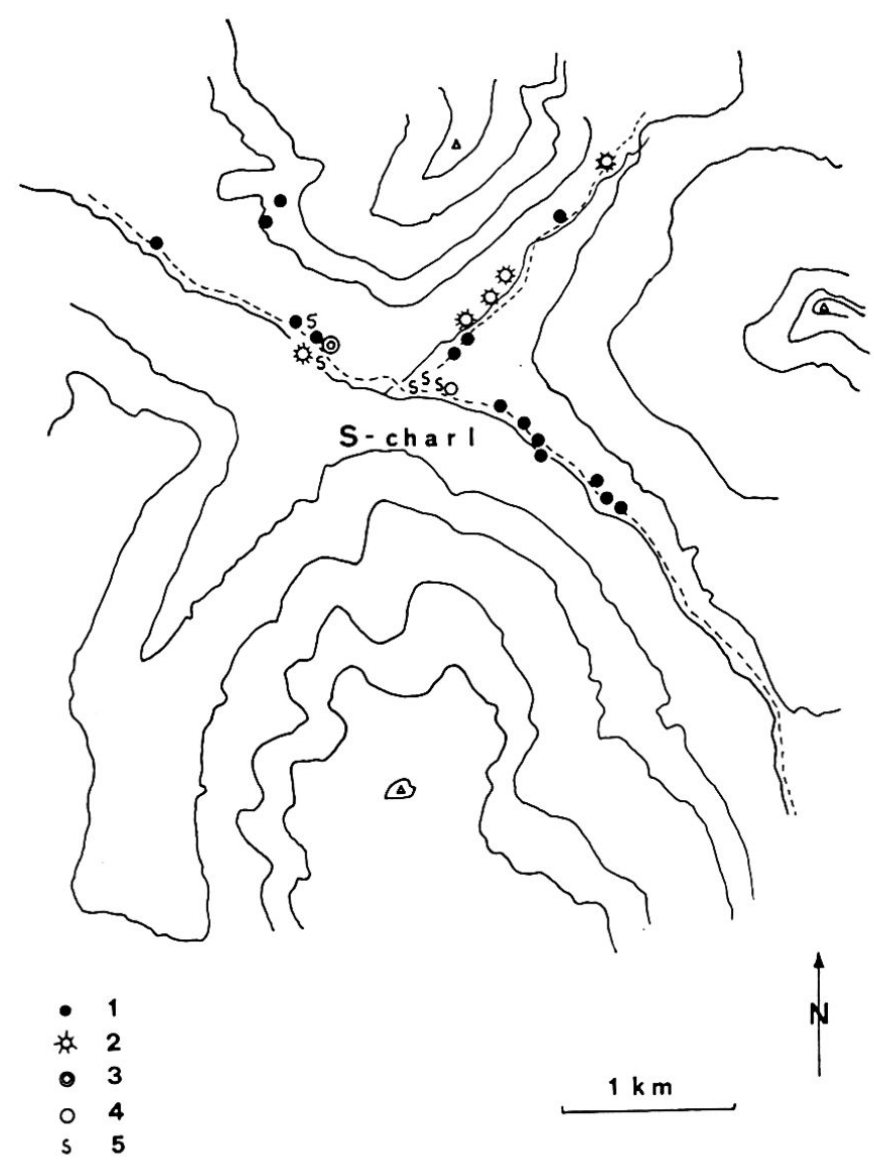

Figur 2. Legende: 1 Kohlenmeiler, 2 Kalkbrennofen, 3 "Schmelzra», 4 vermutete Schmelzanlage, 5 Schlacken 


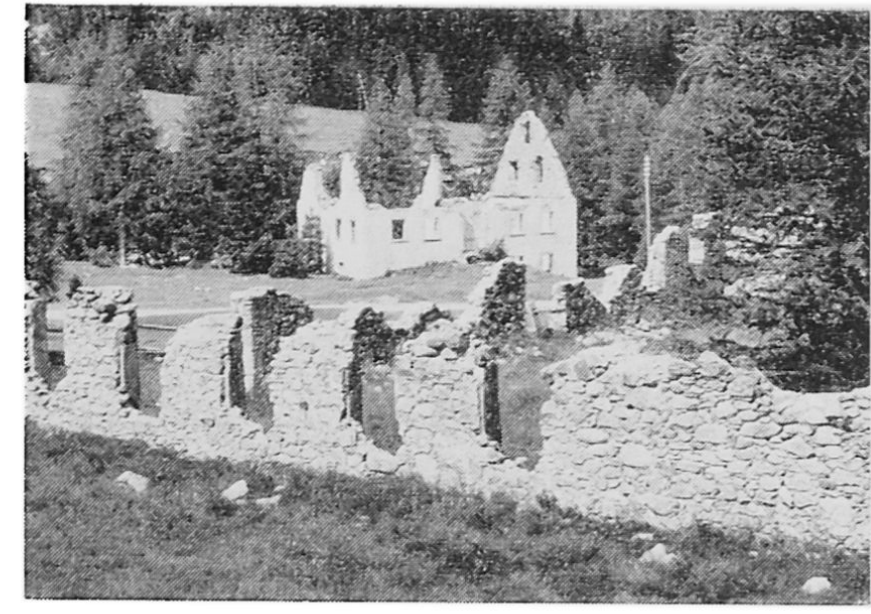

Abbildung 4. Ruinenkomplex «Schmelzra». Im Vordergrund ein Teil von Gebäude A, Raum 1, im Hintergrund Gebäude C

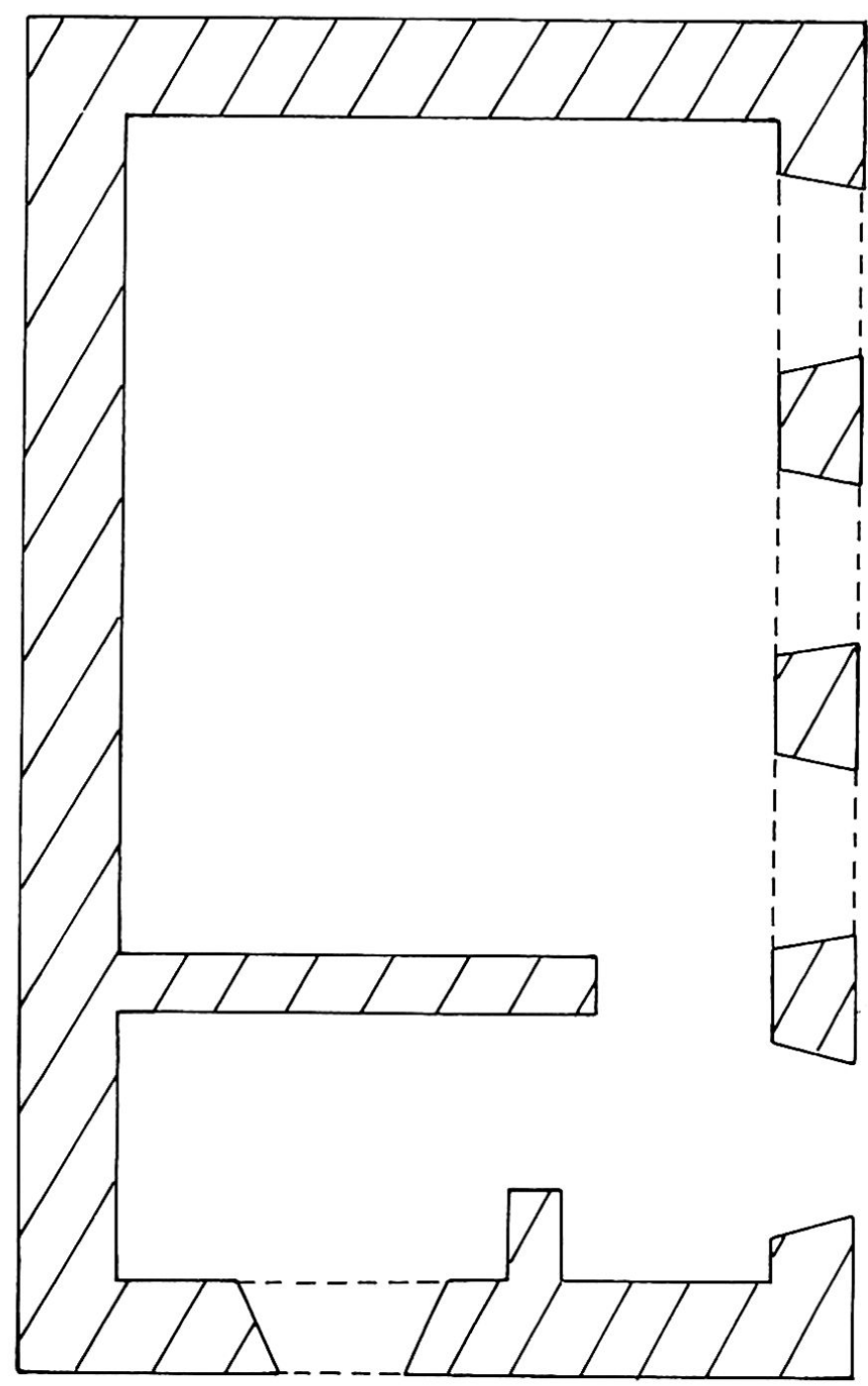

$1 \mathrm{~m}$

Figur 3. Ruine Untermadlein, Grundriß
Der größte Bau nordöstlich der Straße (A) diente in erster Linie zum Pochen und Waschen (Raum 1). Erhalten sind die Mauern des Erdgeschosses (Abb. 4) sowie Teile eines Holzbodens. Ein von der Clemgia abzweigender Kanal (2) führte den Rändern für die Blasebälge und Pochwerke Wasser zu. AnschlieBend an den Raum 1 findet man den Schmelzraum (5) mit einem Kamin (7), während gegen das Gebäude B eine Küche mit Kamin (10) sowie Unterkunftsräume anschließen. Auch das benachbarte Gebäude B erfüllte Wohnfunktionen (Küche 8, Wohnzimmer 6), daneben befand sich hier ein Treibherd (9), ein Schmelzraum (4), und ein Kohlenraum (3).

Das vierstöckige Gebäude C (Abb. 4) wurde auf den Ruinen einer alten Schmelzhütte erbaut. Es enthielt Wohnungen für den Schichtmeister und Knappen sowie eine Probierstube (Laboratorium). Der Bau diente zeitweise auch als Schulhaus.

Etwa $50 \mathrm{~m}$ nordwestlich des Gebäudes B befindet sich nochmals eine Poch- und Schmelzanlage D, bestehend aus drei Räumen. Außer den genannten Schmelzanlagen waren in "Schmelzra» auch zwei Kalkbrennöfen in Betrieb, weitere im Sesvennaund Clemgiatal (vgl. Fig. 2).

\section{Schlußbemerkung}

Während in den aufgelassenen Minen kaum mit bedeutenden Veränderungen zu rechnen ist, sind die Ruinen der Sortier-, Poch- und Schmelzanla-<smiles>C1CC2CC12</smiles>

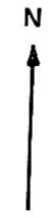

B

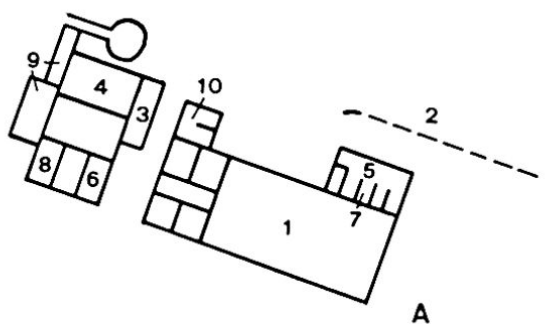

C

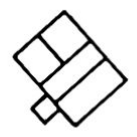

$20 \mathrm{~m}$

Figur 4. Ruinenkomplex «Schmelzra» 
gen sowie der übrigen Bauten teilweise stark im Zerfall begriffen, diejenigen von "Schmelzra» stehen heute unter Denkmalschutz. Es wäre sehr zu begrüßen, wenn die bereits von privater Seite unternommenen Anstrengungen zur Erhaltung dieser wirtschaftshistorisch bedeutenden Überreste weitere Unterstützung erfahren würden. Herr Georg Peer, dem wir zahlreiche wertvolle Hinweise verdanken, hat sich seit Jahrzehnten mit der Erforschung der ganzen Anlagen befaßt, außerdem erste Konservierungsarbeiten geleistet. Hoffentlich bleiben er und seine initiative Gruppe nicht allein. Abschließend möchten wir den aufgeschlossenen und freundlichen Leuten von S-charl für ihre Gastfreundschaft und Hilfe danken, eingeschlossen Herr Pfarrer Gaudenz in Schuls.

\section{Literatur}

Boesch, H., 1936: Der Bergbau am Ofenpaß nördlich der Paßhöhe. Jber. Natf. Ges. Graub. 74, NF. Escher, E., 1935: Erzlagerstätten und Bergbau in Schams, in Mittelbünden und im Engadin. Beitr. $z$. Geol. d. Schweiz., Geotechn. Serie, 18.
Kellerhals, P., 1962: Neue Beobachtungen in den aufgelassenen $\mathrm{Pb}-\mathrm{Zn}$-Bergwerken von S-charl (Unterengadin). Eclogae geologicae Helvetiae vol. 55, Nr. 2.

- 1966: Geologie der nordöstlichen Engadinerdolomiten zwischen Piz San Jon, S-charl und Piz Sesvenna. Diss. Universität Bern.

Parker, R., 1967: Mineralienkunde.

Peer, G., 1968: Las minieras dal Mot Madlain in S-charl. In: Chalender ladin.

- 1970: La fundaria o "Schmelzra» in S-charl. In: Chalender ladin.

Rauch, J. O., 1957: Aus der Geschichte von S-charl. Gästeblatt S-cuol-Tarasp-Vulpera, 12. Jg. Nr. 2.

Schläpfer, D., 1960: Der Bergbau am Ofenpaß. Erg. d. Wissenschaftl. Untersuchung d. schw. Nat.Parks, 7, NF, Nr. 1.

Schleich, K., 1952: Der Bergbau in S-charl. Manuskript.

Adresse des Verfassers:

Dr. Peter Abt

Im Lätt, 8634 Hombrechtikon 